\title{
Educação popular na unidade básica de saúde: relato de experiência
}

\section{Popular education in the basic health unit: experience report \\ Educación popular en la unidad básica de salud: informe de experiencia}

Antônio Lucas Farias da Silva

ORCID: https://orcid.org/0000-0001-8010-1714

Centro Universitário UniFacid, Brasil

E-mail: lucas1992farias@gmail.com

Geísa de Morais Santana

ORCID: https://orcid.org/0000-0001-8008-888X

Universidade Estadual do Piauí, Brasil

E-mail: geisasantana97@gmail.com

Maria Gabriella Nunes Lima

ORCID: https://orcid.org/0000-0002-6872-895X

Centro Universitário UniFacid, Brasil

E-mail: Gabi_nunes_12@hotmail.com

Emanuel Osvaldo de Sousa

ORCID: https://orcid.org/0000-0003-2825-4275 Centro Universitário Unifacid, Brasi E-mail: emanfisio@hotmail.com

Luzia Cleia da Silva

ORCID: https://orcid.org/0000-0002-2387-9068 Universidade Estadual do Piauí, Brasil E-mail: luziaboaventura@gmail.com

Fabriza Maria da Conceição Lopes

ORCID: https://orcid.org/0000-0002-5318-403X Universidade Estadual do Piauí, Brasil E-mail: fabrizalopes55@gmail.com

Abimael de Carvalho

ORCID: https://orcid.org/0000-0002-4393-778X Universidade Estadual do Piauí, Brasil

E-mail: abimaeldecarvalho123@gmail.com

Arlete França Lopes

ORCID: https://orcid.org/0000-0003-2792-7589 Centro Universitário Unifacid, Brasil

E-mail: arlete_franca@outlook.com

Demerval de Pinho Borges Netto

ORCID: https://orcid.org/0000-0002-9529-4130 Centro Universitário Unifacid, Brasil

E-mail: demervalpinho15@outlook.com

Zildenilson da Silva Sousa

ORCID: https://orcid.org/0000-0002-2707-6123

Centro Universitário Maurício de Nassau, Brasil E-mail: zildenilsonsilva@gmail.com

Nayara da Silva Castro

ORCID: https://orcid.org/0000-0001-5827-1266

Faculdade de Ensino Superior do Piauí, Brasil E-mail: nayaracastro09@gmail.com

Crislayde Maria de Sousa

ORCID: https://orcid.org/0000-0001-9752-1416 Universidade Estadual do Piauí, Brasil E-mail: crislaydesousa@gmail.com

Beatriz Santos de Almeida

ORCID: https://orcid.org/0000-0001-8645-5708 União Brasileira de Faculdades, Brasil Wanderson Exxodo de Oliveira Nascimento ORCID: https://orcid.org/0000-0003-0974-8982 Universidade Estadual do Piauí, Brasil E-mail: wandersong5@outlook.com

Heitor Yuri Nogara

ORCID: https://orcid.org/0000-0002-4593-8655 E-mail: heitornogara21@gmail.com 
Amanda Giovanna Ribeiro Macedo

ORCID: https://orcid.org/0000-0002-9162-8500 Centro Universitário Unifacid, Brasil E-mail: amandagior6@gmail.com

Clarice Bezerra

ORCID: https://orcid.org/0000-0003-0457-4442 Universidade Federal do Rio Grande do Norte, Brasil E-mail: claricebezerraa@outlook.com

Maria Andhiara Kaele Feitosa Silva

ORCID: https://orcid.org/0000-0002-8401-8528

Unidade Regional Brasileira de Educação, Brasil E-mail: Andhiarapsi@gmail.com

Luciana Kelly da Silva Fonseca

ORCID: https://orcid.org/0000-0001-8832-5261 Universidade Federal do Piaú, Brasil E-mail: 1.kelly_fonseca@hotmail.com

Lívia Cibelly Rodrigues de Melo

ORCID: https://orcid.org/0000-0001-7810-9776 Universidade Estadual do Piauí, Brasil E-mail: livia.cibelly07@gmail.com

Ana Luiza de Paula Costa

ORCID: https://orcid.org/0000-0002-0114-5485 Universidade Federal do Triângulo Mineiro, Brasil E-mail: analuiza8@outlook.com.br

Isabelle Yasmim Arruda de Andrade

ORCID: https://orcid.org/0000-0002-4361-6353 Universidade Potiguar, Brasil

E-mail: andradeeisabelle@outlook.com

Francineude Nunes Oliveira

ORCID: https://orcid.Org/0000-0001-7821-3274

Faculdade de Ensino Superior do Piauí, Brasil E-mail: Ledapi.2012@hotmail.com

Drieli Ferreira Costa

ORCID: https://orcid.org/0000-0001-6885-5724 Universidade Federal do Triângulo Mineiro, Brasil E-mail: enfdrieli@gmail.com

Gracilene Oliveira da Silva

ORCID: https://orcid.org/0000-0002-8084-4769 Faculdade Santa Luzia, Brasil

E-mail: gracilene@faculdadesantaluzia.edu.br Elzenira da Silva Rodrigues

ORCID: https://orcid.org/0000-0003-0415-216X Faculdade Metropolitana, Brasil E-mail: elzenira2019@gmail.com

Ester Miranda de Sousa

ORCID: https://orcid.org/0000-0003-2319-4840 Faculdade de Ensino Superior do Piaú, Brasil E-mail: mirandaester70@gmail.com

\section{Resumo}

Objetivo: O objetivo deste trabalho é relatar a experiência de um projeto de extensão universitária na realização de oficinas de educação em saúde na sala de espera de uma UBS do interior do Piauí. Método: Trata-se de um estudo descritivo do tipo relato de experiência, realizado no período de janeiro a dezembro de 2017 na Unidade Básica de Saúde Hilda Veras,localizada na cidade de José de Freitas PI. O projeto teve apoio da Universidade Estadual do Piauí (UESPI) e do Conselho Regional de Fisioterapia. O projeto foi composto por acadêmicos da área da saúde e os encontros ocorriam com periodicidade quinzenal com duração de 1 hora por encontro, a escolha dessa unidade justificou-se pelo acesso facilitado para os acadêmicos. Resultados: Foram realizados 22 encontros na UBS. Um ponto importante a se destacar foram às possibilidades produzidas pelas estratégias de acolhimento que propiciaram uma relação bem próxima entre usuários, seus familiares e as equipes duranteas práticas. Verificamos que o acolhimento, com o tempo estava se tornando algo espontâneo e não engessado ou tímido, como foram nos primeiros instantes. Isso foi possível evidenciar pelo fato de que algumas pessoas da comunidade já passavam a reconhecer os estudantes do projeto, e assim, procurálos para informações, ajuda e até mesmo uma conversa, afim de saber o papel deles no local, o que foi bastante interessante. Conclusão: A partir das experiências no projeto de extensão foi possível promover reflexões sobre a importância do acolhimento nas Unidades Básicas de Saúde, como também sobre a importância da Educaçãoem Saúde. Além de experiências enriquecedoras de trocas de saberes com os grupos, tambémfoi possível aprender mais sobre as metodologias ativas e de acolhimento que as políticas públicas preconizam.

Palavras-chave: Promoção da saúde; Sistema Único de Saúde; Centros de saúde. 


\begin{abstract}
Objective: The objective of this work is to report the experience of a university extension project in carrying out health education workshops in the waiting room of a UBS in the interior of Piauí. Method: This is a descriptive study of the experience report type, carried out from January to December 2017 at the Hilda Veras Basic Health Unit, located in the city of José de Freitas PI. The project was supported by the State University of Piauí (UESPI) and the Regional Council of Physiotherapy. The project was composed of academics from the health area and the meetings took place every fortnight, lasting 1 hour per meeting, the choice of this unit was justified by the easy access for the academics. Results: 22 meetings were held at UBS. An important point to be highlighted were the possibilities produced by the welcoming strategies that provided a very close relationship between users, their families and the teams during the practices. We found that the host, with time, was becoming something spontaneous and not plastered or shy, as it was in the first moments. This was evidenced by the fact that some people in the community already started to recognize the project's students, and thus, look for them for information, help and even a conversation, in order to know their role in the place, which was quite a lot. interesting. Conclusion: Based on the experiences in the extension project, it was possible to promote reflections on the importance of welcoming in Basic Health Units, as well as on the importance of Health Education. In addition to enriching experiences of knowledge exchange with the groups, it was also possible learn more about the active and welcoming methodologies that public policies advocate.
\end{abstract}

Keywords: Health promotion; Unified Health System; Health centers.

\title{
Resumen
}

Objetivo: El objetivo de este trabajo es reportar la experiencia de un proyecto de extensión universitaria en la realización de talleres de educación en salud en la sala de espera de una UBS en el interior de Piauí. Método: Se trata de un estudio descriptivo del tipo relato de experiencia, realizado de enero a diciembre de 2017 en la Unidad Básica de Salud Hilda Veras, ubicada en la ciudad de José de Freitas PI. El proyecto fue apoyado por la Universidad Estatal de Piauí (UESPI) y el Consejo Regional de Fisioterapia. El proyecto estuvo integrado por académicos del área de salud y las reuniones se realizaron quincenales, con una duración de 1 hora por reunión, la elección de esta unidad se justificó por el fácil acceso para los académicos. Resultados: se realizaron 22 reuniones en la UBS. Un punto importante a destacar fueron las posibilidades que produjeron las estrategias de acogida que proporcionaron una relación muy estrecha entre los usuarios, sus familias y los equipos durante las prácticas. Descubrimos que el anfitrión, con el tiempo, se fue convirtiendo en algo espontáneo y no enyesado ni tímido, como en los primeros momentos. Esto fue evidenciado por el hecho de que algunas personas de la comunidad ya comenzaron a reconocer a los estudiantes del proyecto, y así, buscarlos para información, ayuda e incluso una conversación, para conocer su rol en el lugar, que era bastante interesante. Conclusión: A partir de las experiencias del proyecto de extensión, se logró promover reflexiones sobre la importancia de la acogida en las Unidades Básicas de Salud, así como sobre la importancia de la Educación para la Salud. Además de enriquecer las experiencias de intercambio de conocimientos con los grupos, También fue posible conocer más sobre las metodologías activas y acogedoras que propugnan las políticas públicas.

Palabras clave: Promoción de la salud; Sistema único de Salud; Centros de salud.

\section{Introdução}

A Educação Popular em Saúde (EPS) pode ser entendida como um modo particular de reconhecer e enfrentar os problemas de saúde, por meio do diálogo com as classes populares, o respeito às suas culturas, o reconhecimento dos seus saberes como válidos e tendo como substrato o corpo teórico da Educação Popular, formulada por Paulo Freire no Brasil (Alves, 2016). Dessa maneira, a EPS constitui um conjunto de práticas e saberes populares tradicionais que, de acordo com a Política Nacional de Educação Popular em Saúde (PNEP-SUS), mostra-se como um caminho capaz de contribuir com metodologias, tecnologias e saberes para a constituição de novos sentidos e práticas no âmbito do SUS (Pinheiro, 2017; Vasconcelos, 2016).

A proposta freiriana não é que a contemplação seja com visão de algo que só exista nas ideias, ou que estejam em equilíbrio, mas é aquela que visa a transformação, sem que haja acomodação ou descaso com a situação que se apresenta (Oliveira, et al., 2021; Paro et al., 2019). E é de forma semelhante ao que foi proposto por Freire, que devem ser planejadas as estratégias educativas em saúde, visando a confluência de entendimentos teóricos e práticos (Oliveira \& Cota, 2018).

A EPS busca, além da construção de uma consciência sanitária capaz de reverter o quadro de saúde da população, a intensificação da participação popular, contribuindo para a promoção da saúde (Gomes \& Merhy, 2011). Ela parte do pressuposto de que o educando possui um saber prévio, construído em sua história de vida, sua prática social e cultural, que lhe serve de ponto de partida para a aquisição de novos conhecimentos. A educação se constitui como um processo de busca e de invenção ou reinvenção que parte da ação e da reflexão do homem sobre o mundo, para transformá-lo (Bornstein, 2007). 
Nesse contexto, a Atenção Básica (AB) configura o primeiro nível de atenção à saúde, englobando um conjunto de ações de saúde, nos âmbitos individual e coletivo, visando à promoção de saúde, prevenção de agravos, tratamento, reabilitação e recursos de manutenção à saúde. A proposta da $\mathrm{AB}$ é ser a porta de entrada e centro de comunicação da Rede de Atenção à Saúde, por meiode Unidades Básicas de Saúde (UBS) e Estratégia de Saúde da Família (ESF) (Brasil, 2017).

As UBS surgiram na década de 1980 no contexto de sistematização dos serviços de saúde, com a finalidade de alcançar resolutividade nas questões referentes à saúde e melhor eficácia nos tratamentos. Por isso podem ser consideradas como uma das geradoras do SistemaÚnico de Saúde (SUS). O SUS, por sua vez, ampliou concepções sobre processos de saúde e de adoecimento, por considerar seus fatores condicionantes e determinantes, como alimentação, saneamento básico, moradia, meio ambiente, trabalho, renda e transporte (Brasil, 2017).

A busca de um novo modelo assistencial centrado na Atenção Primária à Saúde (APS) decorre de um momento histórico social, em que o modelo tecnicista, hospitalocêntrico não atende mais à emergência das mudanças do mundo moderno e, consequentemente, às necessidades de saúde dos usuários. Nesse sentido, a Estratégia de Saúde da Família (ESF) se apresenta como um modelo de atenção dos serviços de saúde, tendo a família no centro de atenção, incorporando um novo olhar no processo de intervenção e buscando a implementação de ações preventivas (Ministério da Saúde, 2014).

A APS é o primeiro nível de assistência no sistema e tem como seus atributos essenciais o acesso de primeiro contato do indivíduo com o sistema de saúde, a continuidade e a integralidade da atenção e coordenação da atenção dentro do sistema. Além de outros três atributos derivados: a orientação familiar e comunitária e a competência cultural. A partir disso, tem-se uma perspectiva de APS acolhedora, resolutiva que avança na gestão e coordenação do cuidado do usuário na rede de atenção à saúde (RAS) (Starfield, 2002).

A Estratégia Saúde da Família (ESF) configura um modelo assistencial em que as práticas devem estar orientadas pelos determinantes do processo saúde-doença, considerando o indivíduo e o seu contexto familiar, social, econômico e cultural, bem como a realização das ações de vigilância em saúde e promoção da saúde. A ESF deve ser operacionalizada por equipes multiprofissionais, em Unidades Básicas de Saúde (UBS), em um território definido (Fertonani, et al., 2015).

As UBS, por serem peças fundamentais da $\mathrm{AB}$, integram a ESF como prioridade para sua organização, e é nessa articulação entre UBS e ESF que se obedece e trabalha em favor dos princípios do SUS. As UBS focam a atenção de uma população específica que está em um território definido. Assumem, portanto, a responsabilidade sanitária e o cuidado a essas pessoas, e trabalham considerando as particularidades existentes no território em que elas vivem (Brasil, 2017).

A metodologia de ensino que emprega a abordagem humanista é aquela com foco no aprendiz, que é o protagonista e o condutor da trilha pedagógica, onde ocorrerá atribuição de novo significado a acontecimentos através da mudança de sua visão de mundo, com consequente alteração integral de caráter e de personalidade (Brasil, 2013). Em grupos, pode ser realizada através de rodas de conversa, compreendida como constituição de momentos mediados por uma pessoa, que normalmente é o profissional de saúde, oportunos para as trocas de conhecimentos a respeito de determinado assunto, numa relação entre iguais, ou seja, dois seres humanos com seus direitos naturais e que colaboram por perceberem vantagem nisso (Oliveira \& Leite, 2011; Nascimento \& Baduy, 2021).

A partir da conjuntura anteriormente apresentada, o objetivo deste trabalho é relatar a experiência de um projeto de extensão universitária na realização de oficinas de educação popular em saúde na sala de espera de uma Unidade Básica de Saúde do interior do Piauí.

\section{Metodologia}

Trata-se de um estudo descritivo, com abordagem qualitativa, do tipo relato de experiência (Daltro \& Faria, 2019), realizado no período de janeiro a dezembro de 2017 na Unidade Básica de Saúde Hilda Veras, localizada na cidade de José de 
Freitas PI. O projeto teve apoio da Universidade Estadual do Piauí (UESPI) e do Conselho Regional de Fisioterapia. O projeto foi composto por acadêmicos da área da saúde e os encontros ocorriam com periodicidade quinzenal com duração de 1 hora por encontro, a escolha dessa unidade justificou-se pelo acesso facilitado para os acadêmicos

O relato de experiência, segundo o Instituto de Ciências da Vida, da Universidade Federal de Juiz de Fora (2017) é um texto que descreve precisamente uma dada experiência que possa contribuir de forma relevante para sua área de atuação. Segundo este instrutivo de elaboração de relatos de experiência, ele apresenta as motivações ou metodologias para as ações tomadas na situação e as considerações/impressões que a vivência trouxe àquele (a) que a viveu.

Inicialmente, foi realizado um acolhimento pelos acadêmicos que explicaram o objetivo do projeto e os procedimentos a serem seguidos, logo após, o convite foi feito aos usuários para participação das atividades. O público alvo era a população em geral, que aguardavam o atendimento médico e do dentista, o tema de cada encontro foi escolhido de acordo com o público específico de cada dia na UBS, pois cada dia da semana eram atendidas pessoas de diferentes faixas etária, além disso, existiam dias exclusivos para pacientes com comorbidades como, hipertensão e diabetes. Ao término de cada encontro era realizado uma tempestade de ideias com os usuários, para assim fazer um levantamento de temas que os pacientes gostariam que fossem abordados nos encontros.

Os encontros ocorriam durante a espera dos atendimentos das consultas médicas e odontológicas, as mesmas aconteciam de forma simultânea, fazendo com que tivesse uma grande aglomeração de pacientes, facilitando a participação de mais pessoas no projeto. Durante a ação, foram realizadas rodas de conversa em uma sala de espera reservada na unidade de saúde com intuito de dinamizar, instigar e facilitar o vínculo entre os acadêmicos da ação e participantes do projeto, para que o momento fosse de partilha e aprendizado para ambas as partes.

As Rodas de Conversa foram utilizadas como uma metodologia participativa com a população em geral atendidas na referida unidade, para tanto foram utilizadas metodologias ativas e participativas que estimulavam e valorizavam o contato e o saber dos atores envolvidos. A roda de conversa era organizada em momentos, desde acolhimento, exposição dialogada, e interação com as participantes, com espaço para sugestões, dúvidas, relato de experiências e discussão coletiva.

\section{Resultados e Discussão}

Humanizar na atenção à saúde é levar em consideração as vivências e valores das pessoas de forma individualizada (Rech, 2003). A ação desenvolvida em sala de espera foi ao encontro da Política Nacional de Humanização do SUS, proporcionando ao usuário a troca e construção de saberes e uma escuta qualificada sobre questões da saúde. Além disso, permitiu aos acadêmicos o contato com outros profissionais da rede e levou o aluno, juntamente com a equipe do centro de saúde, a refletir sobre as necessidades locais, viabilizando a elaboração e organização de ações de promoção de saúde voltadas para as necessidades e interesses da população em geral.

Um ponto importante a se destacar foram às possibilidades produzidas pelas estratégias de acolhimento que propiciaram uma relação bem próxima entre usuários, seus familiares e as equipes durante as práticas. Verificou-se que o acolhimento, com o tempo estava se tornando algo espontâneo e não engessado ou tímido, como foram nos primeiros instantes. Isso foi possível evidenciar pelo fato de que algumas pessoas da comunidade já passavam a reconhecer os estudantes do projeto, e assim, procurá-los para informações, ajuda e até mesmo uma conversa, afim de saber o papel deles no local, o que foi bastante interessante.

A metodologia da problematização tenciona o modelo tecnocientífico, com seus processos de análise da realidade e de construção do conhecimento. Pode mesmo vir a reorientar o entendimento sobre adoecimento, pois garante maior visibilidade às questões sociais, culturais e psicológicas do indivíduo/paciente. Ademais, facilita a ruptura de paradigmas em educação, possibilitando formar profissionais em Saúde com novos pensamentos (Darius \& Lopes, 2017). 
Segundo Assis et al. (2020), a reflexão crítica, o diálogo e a construção compartilhada do conhecimento representam ferramentas que propiciam o encontro entre a cultura popular e a científica e contribuem para a construção de um pensamento social em saúde. A disponibilidade de escuta e fala dos atores que se põem em relação possibilita uma visão de saberes e práticas diferentes, convivendo em situações de reciprocidade e cooperação. Através do diálogo entre estes saberes, os profissionais de saúde adquirem formação comprometida com as questões sociais, não somente pela mudança de atitudes e comportamentos, mas principalmente pelo engajamento ativo nas lutas por direitos e comprometimentos com posturas acolhedoras e de construção da autonomia das pessoas e dos grupos sociais. Os usuários ressignificam o direito à saúde, na luta pela inclusão social (Vasconcelos, 2004).

O primeiro encontro foi apenas com os funcionários e profissionais da UBS, com intuito de fazer o reconhecimento do local, saber os horários dos atendimentos e a forma que ocorriam. A partir do segundo encontro foram elaboradas estratégias e temas específicos para o público alvo do dia, representado conforme o Quadro 1.

Quadro 1: Temas e práticas desenvolvidas nos encontros na Unidade Básica de Saúde.

\begin{tabular}{|c|c|}
\hline O QUE É SAÚDE? & $\begin{array}{l}\text { Foi feita a construção de cartazes sobre como ter uma boa saúde, assim como, } \\
\text { ocorreu o compartilhamento de conhecimentos, sempre buscando trabalhar em } \\
\text { grupo. }\end{array}$ \\
\hline $\begin{array}{l}\text { ACIDENTE VASCULAR } \\
\text { ENCEFÁLICO (AVE) }\end{array}$ & $\begin{array}{l}\text { Ocorreu uma roda de conversa para debater sobre o tema, como também, foi } \\
\text { feito um jogo sobre Mitos e Verdades. Ao final do encontro foi entregue um } \\
\text { panfleto com informações sobre os sintomas, as causas e o tratamento para o } \\
\text { AVE. }\end{array}$ \\
\hline DIABETES & $\begin{array}{l}\text { Houve discussão em grupo sobre o tema, realização de atividades físicas e sua } \\
\text { importância para a saúde. Ao término do encontro foi entregue panfletos sobre } \\
\text { as causas, os tipos e o tratamento para a doença. }\end{array}$ \\
\hline SAÚDE DO TRABALHADOR & $\begin{array}{l}\text { Foi feita uma roda de conversa para discussão sobre o tema, logo após, os } \\
\text { usuários participaram de um jogo de perguntas e respostas para esclarecer suas } \\
\text { principais dúvidas relacionadas ao trabalho. }\end{array}$ \\
\hline HIPERTENSÃO & $\begin{array}{l}\text { Iniciou-se com uma roda de conversa para discutir sobre o tema, foi feito um } \\
\text { jogo de perguntas e respostas, logo após, os acadêmicos repassaram as principais } \\
\text { informações sobre o tema, ao final do encontro foi realizado a entrega de } \\
\text { panfletos com as principais informações sobre a doença. }\end{array}$ \\
\hline SÁUDE DA CRIANÇA & $\begin{array}{c}\text { As crianças foram divididas em grupos e desafiadas a criar um cartaz com as } \\
\text { principais informações sobre saúde, ao término do cartaz, todos os grupos } \\
\text { fizeram a apresentação. Ao final do encontro os acadêmicos observaram } \\
\text { pontos positivos decada grupo e acrescentaram outras informações } \\
\text { importantes sobre a saúde da criança. }\end{array}$ \\
\hline SAÚDE DO IDOSO & $\begin{array}{l}\text { Primeiramente, foi feita uma roda de conversa para discussão sobre o tema, ao } \\
\text { final do encontro os acadêmicos abordaram sobre algumas temáticas que } \\
\text { influenciam naqualidade de vida do idoso, como, alimentação, atividade física } \\
\text { e a importância do sono. }\end{array}$ \\
\hline BOA POSTURA & $\begin{array}{c}\text { Inicialmente, os acadêmicos falaram sobre os benefícios da boa postura e sua } \\
\text { importância para a saúde. Foi criado um mural com as posturas CORRETAS } \\
\text { X ERRADAS, o mesmo ficou exposto na UBSpara que todos pudessem ter } \\
\text { acesso às informações sobre uma boa postura. }\end{array}$ \\
\hline
\end{tabular}

Fonte: Autores.

Após o término de todos os encontros foram distribuídos panfletos educativos, com intuito de fortalecer e concretizar os conhecimentos repassados pelos acadêmicos. Vale ressaltar que o formato de rodas de conversa, através da dialética, permite ao pesquisador se inserir como sujeito da pesquisa pela participação na conversa $\mathrm{O}$ uso das metodologias ativas de ensino e aprendizagem promove a socialização dos atores envolvidos na aprendizagem, o compartilhamento de conhecimentos, a reflexão sobre os conteúdos teóricos e práticos, e acima de tudo, torna o processo educativo mais dinâmico e participativo (Carraro, et al., 2011).

Projetos de extensão como esse, são uma excelente alternativa para melhorar a qualidade de vida das pessoas, além de 
permitir uma troca de conhecimentos entre a população e os acadêmicos, assim como, permitir que os acadêmicos tivessem o contato prévio com as principais necessidades da população de forma geral.

Dentre os efeitos produzidos pala experiência, sublinha-se o impacto da educação popular em saúde nos discentes do projeto. Usualmente, a formação concentra-se na força do conhecimento acadêmico e, na maior parte do tempo, são raros os momentos de oitiva da população/comunidade. Os docentes tiveram a oportunidade de observar a atuação de seus alunos, o que foi inovador para o grupo.

O movimento de Educação Popular em Saúde incentiva a prática educativa, em uma perspectiva horizontal da relação trabalhador-usuário, proporcionando trocas interpessoais (Junges, et al., 2017). O interesse da população usuária, pelo diálogo, busca compreender o saber popular. Esse método reconhece o usuário como sujeito capaz de fornecer uma interlocução dialógica com o serviço de saúde e de desenvolver uma análise crítica sobre a realidade, possibilitando implementar estratégias de luta e de enfrentamento (Santos \& Araujo, 2020).

O protagonista das ações de EPS deve buscar, no processo de aprendizagem das práticas, uma reflexão profunda e avaliação crítica a respeito das situações-limite de suas práticas, e conhecer que caminhos podem ser construídos para superálas. Isso deve ser pensado com o objetivo de qualificação e construção de alternativas para os principais desafios colocados no SUS (Cruz, et al., 2020).

O objetivo da Roda de Conversa é estimular a construção da autonomia dos sujeitos por meio da problematização, da troca de informações e da reflexão para a ação (Melo \& Cruz, 2014). A roda de conversa na gestão em saúde é uma forma de buscar construir espaços de partilha, confronto de ideias e entendimento baseado na liberdade de diálogo entre os participantes na intenção de se afirmar com uma alternativa importante para ampliar o grau de corresponsabilidade das ações na produção de saúde (Farias et al., 2008).

A educação em saúde é, muitas vezes, entendida como um modo de fazer as pessoas do povo mudarem seus hábitos para assimilarem práticas higiênicas e recomendações médicas que evitariam o desenvolvimento de um conjunto de doenças. Entretanto, para os autores que se baseiam na educação popular, educar para a saúde é justamente ajudar a população a compreender as causas dessas doenças e a se organizar para superá-las (Vasconcelos, 1997).

Para Gadotti (2017), as atividades de extensão universitária buscam aproximar diversos setores da sociedade da comunidade acadêmica, mediante ações inter-relacionadas realizadas em determinados territórios por meio de elaborações de estratégias de abordagem e de soluções de problemáticas sociais. Foi possível aprender muitas coisas a respeito das realidades e como as equipes lidam com as prioridades, tendo como base o entendimento das distintas realidades e particularidades da vida cotidiana que envolve os indivíduos e suas famílias.

\section{Conclusão}

A partir das experiências no projeto de extensão foi possível promover reflexões sobre a importância do acolhimento nas Unidades Básicas de Saúde, como também, sobre a importância da Educação popular em Saúde. Além de experiências enriquecedoras de trocas de saberes com os grupos, também foi possível aprender mais sobre as metodologias ativas e de acolhimento que as políticas públicas preconizam. Assim, a roda de conversa mostrou-se como uma ótima ferramenta que possibilita a troca de conhecimentos, de saberes populares, bem como, estimula a autonomia dos usuários em relação a sua saúde.

Conclui-se que a participação da comunidade na construção dos momentos crítico-reflexivos, permite que eles se sintam como parte do processo, através do espaço acolhedor. Assim, sugere-se mais estudos sobre a abordagem da educação popular em saúde na comunidade, com novos temas, principalmente sobre a pandemia do novoconavírus. 


\section{Referências}

Alves, S. R., Alves, A. O. \& Assis, M. C. S. (2016). Educação Popular em Saúde como estratégia à adesão na realização do exame colpo citológico. Ciênc. Cuid. Saúde. 15(3), 570-574.

Assis, V. D., Sabino, R. N., Neves, V. N. S., Santos, S. T. A., Andrade, C. A. C. \& Rodrigues, J. M. C. (2020). A vocação transformadora da educação popular em saúde. Research, Society and Development. 9(12), 1-19. http://dx.doi.org/10.33448/rsd-v9i12.11296.

Brasil. Ministério da saúde. Política Nacional de Educação Popular em Saúde no Sistema Único de Saúde. (2013). Ministério da Saúde. https://bvsms.saude.gov.br/bvs/saudelegis/gm/2013/prt2761_19_11_2013.html

Brasil. (2017). Ministério da Saúde: Portaria no 2.436, de 21 de setembro de $2017 . \quad$ Autor. 2017. http://bvsms.saude.gov.br/bvs/saudelegis/gm/2017/prt2436_22_09_2017.html

Bornstein, V. J. (2007). O agente comunitário de saúde na mediação de saberes [tese]. Rio de Janeiro (RJ): Escola Nacional de Saúde Pública Sergio Arouca.

Carraro, T. E., Prado, M. L., Silva, D. G. V., Radünz, V., Kempfer, S. S. \& Sebold, L. F. (2011). Socialização como processo dinâmico de aprendizagem na enfermagem. Uma proposta na metodologia ativa. Investigación y Educación en Enfermería, 29 (2), 248-254.

Cruz, P. J. S. C., Silva, M. R. F. \& Pulga, V. L. (2020). Educação Popular e Saúde nos processos formativos: desafios e perspectivas. Interface (Botucatu). 24(1), 1-15. http://dx.doi.org/10.33448/rsd-v9i12.11296.

Daltro, M. R. \& Faria, A. M. (2019). Relato de experiência: Uma narrativa científica na pós-modernidade. Estudos e Pesquisas em Psicologia, 19(1), 223-239.

Darius, R. P. P. \& Lopes, B. J. S. (2017). O uso da metodologia da problematização para o desenvolvimento de projeto integrador no curso de pedagogia. Revista Ibero-Americana de Estudos em Educação,12 (2), 983-1004.

Farias, A. M., Rocha, A. A. A. S. \& Neves, N. (2008). A. Roda de conversa: contribuição da educação popular em saúde à gestão participativa do sus no município de Paudalho [tese]. Recife.

Fertonani, H. P., Pires, D. E. P., Biff, D. \& Scherer, M. D. A. (2015). Modelo assistencial em saúde: conceitos e desafios para a atenção básica brasileira. Ciênc. saúde coletiva. 20(6), $1869-1878$.

Gadotti, M. (2017). Extensão universitária: para quê. Instituto Paulo Freire.

Gomes, L. B. \& Merhy, E. E. (2011). Compreendendo a educação popular em saúde: um estudo na literatura brasileira. Cad Saude Publica, 27(1), 7-18.

Instituto de Ciências da Vida (2017). Departamento de Nutrição. Instrutivo para Elaboração de Relato de Experiência. Estágio em Nutrição em Saúde Coletiva. Universidade Federal de Juiz de Fora - UFJF - Campus Governador Valadares.

Junges, J. R., Barbiani, R., Soares, N. A., Fernandes, R. B. P. \& Lima, M. S. (2017). Saberes em Paulo Freire: Educação Popular em Saúde na procura por uma "Pedagogia da Esperança" e seus entrelaços com o processo emancipatório de LGBT. Revista Rede de Cuidados em Saúde, 11(1), 1-12.

Melo, M. C. H. \& Cruz, G. C (2014). Roda de conversa: uma proposta metodológica para a construção de um espaço de diálogo no ensino médio. Imagens da Educação, 4 (2), 31-39.

Ministério Da Saúde (2014). Secretaria de Atenção à Saúde, Implantação das Redes de Atenção àSaúde e outras estratégias da SAS/Ministério da Saúde. Secretaria de Atenção à Saúde. Brasília: Ministério da Saúde.

Nascimento, A. K. C. \& Baduy, R. S (2021). Simulação, oficina e roda de conversa: estratégias de aprendizagem ativa na saúde. Revista Educação em Debate. 43(84), 152-167.

Oliveira, L. M. P. \& Leite, M. T. M. (2011). Concepções Pedagógicas. Módulo Pedagógico. Especialização em Saúde da Família - Modalidade a Distância. UNA-SUS UNIFESP. https://www.unasus.unifesp.br/biblioteca_virtual/esf/1/modulo_pedagogico/Unidade_1.pdf

Oliveira, M. F. \& Cota, L. G. S. (2018). A pedagogia freiriana nas práticas de educação em saúde. Diversitates International Journal, 10(1), 46-58.

Oliveira, M. F., Spósito, P. A. F., Lima, C. S. A. \& Cupertino, M. C. (2021). Roda de conversa em um ambulatório público: o papel da atenção primária na educação popular em saúde. Research, Society and Development, 10(13), 1-8. http://dx.doi.org/10.33448/rsd-v10i13.21256.

Paro, C. A., Ventura, M. \& Silva, N. E. K. (2019). Paulo Freire e o inédito viável: esperança, utopia e transformação na saúde. Trabalho, Educação e Saúde, $18(1), 1-22$.

Pinheiro, B. C. \& Bittas, C. M. L. (2017). Práticas de Educação Popular em Saúde na atenção primária: uma revisão integrativa. Cinergs, 18(1), 1-6.

Rech, C. M. F. (2003). Humanização hospitalar: o que pensam os tomadores de decisão a respeito? [dissertação]. Faculdade de Saúde Pública, Universidade de São Paulo.

Santos, J. B. \& Araujo, E. J. M. (2020). A educação do campo no campo da educação popular. Revista de Educação Popular. 18(3), 56-73.

Starfield (2002). Atenção primária: equilíbrio entre necessidades de saúde, serviçose tecnologia. Unesco.

Vasconcelos, E. M., Cruz, P. J. S. C. \& Prado, E.V. (2016). A contribuição da Educação Popular para a formação profissional em saúde. Interface -Comunicação, Saúde, Educação [online], 20(59), 835-838. http://dx.doi.org/10.1590/1807-57622016.0767.

Vasconcelos, E. M. (2004). Educação popular: de uma prática alternativa a uma estratégia de gestão participativa das Políticas de Saúde. Physis. 14 (1), 67-83.

Vasconcelos, E. M. (1997). Educação popular nos serviços de saúde. (3a ed.), Editora Hucitec. 